\title{
Corrigendum
}

\section{Corrigendum to "A New Iterative Algorithm Based on Correction of Sensitivity Matrix for Electrical Resistance Tomography"}

\author{
Yutong Chen $\mathbb{D}^{\mathbb{1}},{ }^{1,2}$ Yan Han $\mathbb{D}^{1},{ }^{1}$ Wuqiang Yang $\mathbb{D}^{3},{ }^{3}$ and Kun Li $\mathbb{D}{ }^{1,4}$ \\ ${ }^{1}$ Shanxi Key Lab of Signal Capturing and Processing, North University of China, Taiyuan 030051, China \\ ${ }^{2}$ Department of Electrical Engineering and Automation, Shanxi Polytechnic College, Taiyuan 030006, China \\ ${ }^{3}$ Department of Electrical and Electronic Engineering, University of Manchester, Manchester M13 9PL, UK \\ ${ }^{4}$ School of Chemical and Process Engineering, University of Leeds, Leeds LS2 9JT, UK
}

Correspondence should be addressed to Yutong Chen; cyt_nuc@163.com

Received 28 January 2020; Accepted 29 January 2020; Published 28 May 2020

Copyright ( $\odot 2020$ Yutong Chen et al. This is an open access article distributed under the Creative Commons Attribution License, which permits unrestricted use, distribution, and reproduction in any medium, provided the original work is properly cited.

In the article titled "A New Iterative Algorithm Based on Correction of Sensitivity Matrix for Electrical Resistance Tomography" [1], there was an error in the "Introduction" section, where "ERT has been widely used for chemical [2], food processing [3], biology [4], geology [5], and other industrial applications [6]" should be corrected to "ERT has been widely used for chemical [2], thin films characterization [3], biology [4], geology [5], and other industrial applications [6]."

\section{References}

[1] Y. Chen, Y. Han, W. Yang, and K. Li, "A New iterative Algorithm based on correction of sensitivity Matrix for electrical resistance Tomography," Mathematical Problems in Engineering, vol. 2019, Article ID 6384132, 15 pages, 2019. 